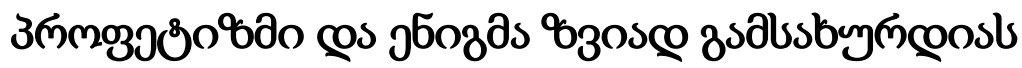 उmģosðo
}

jomos 5s5s

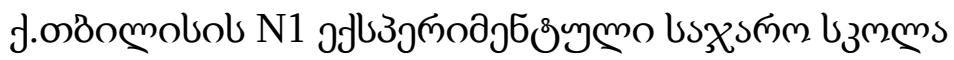
askf̧sз

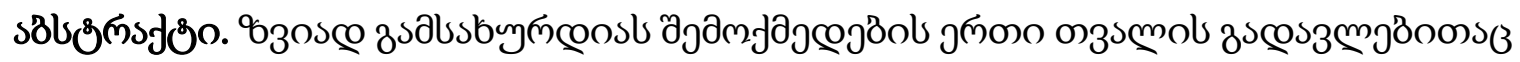

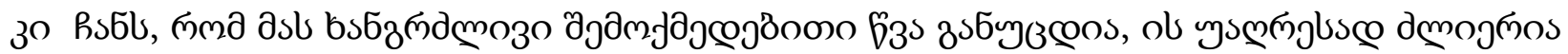

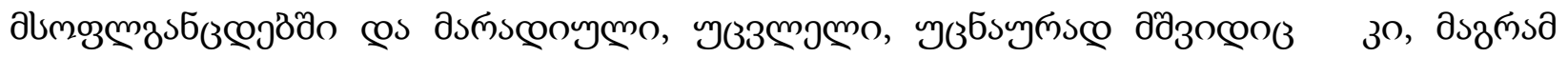

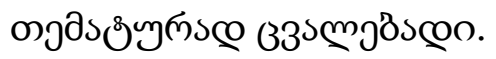

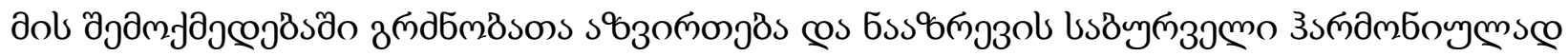

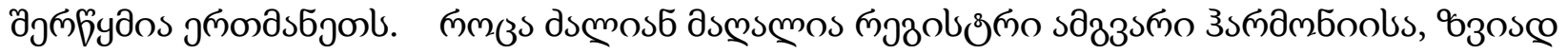

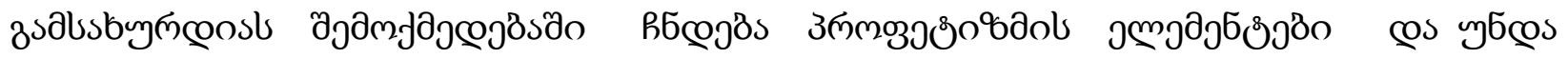

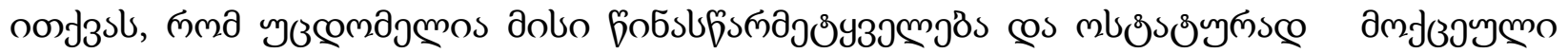

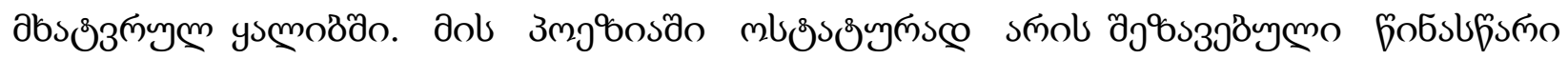

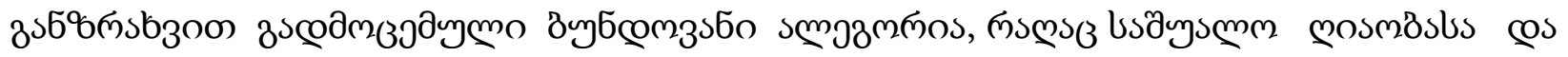

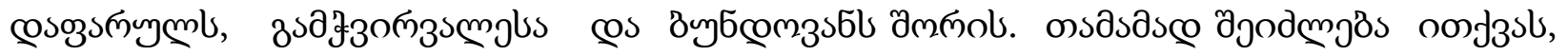

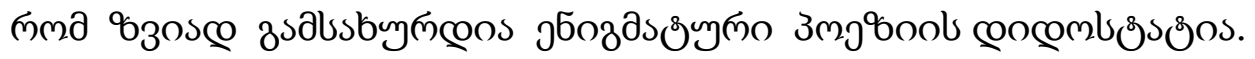

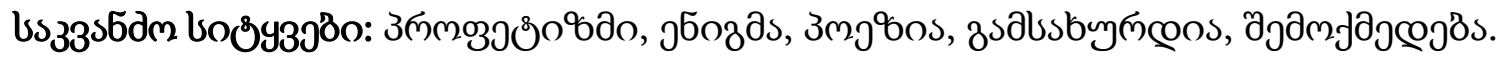

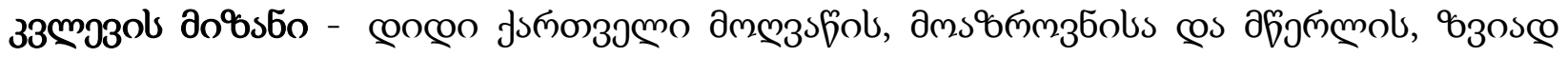

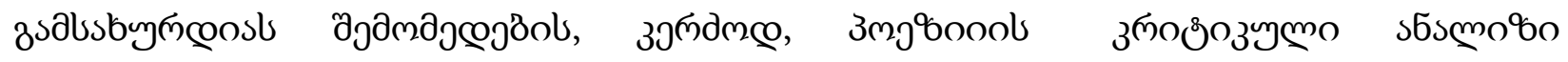

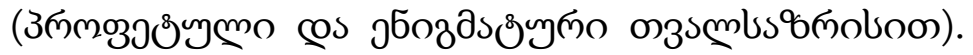

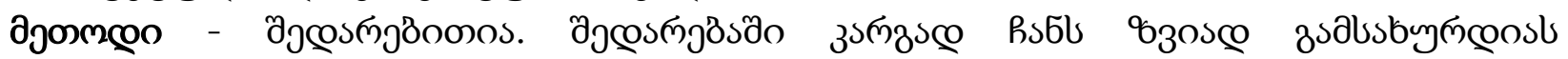

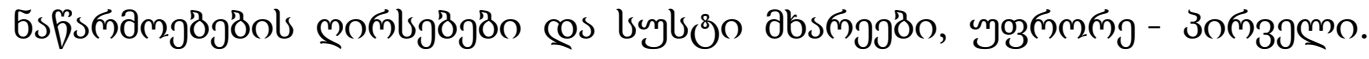

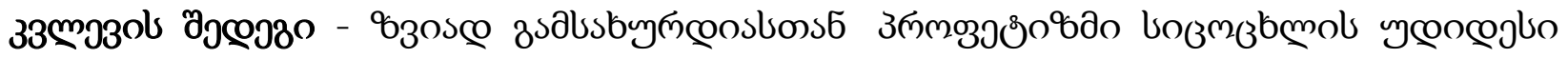

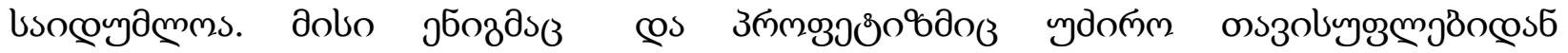
дмдсообьпомды.

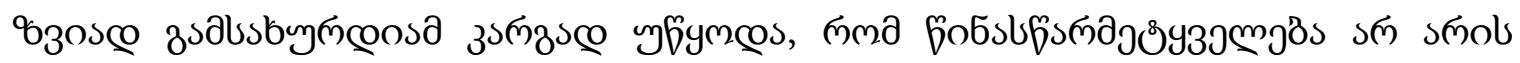

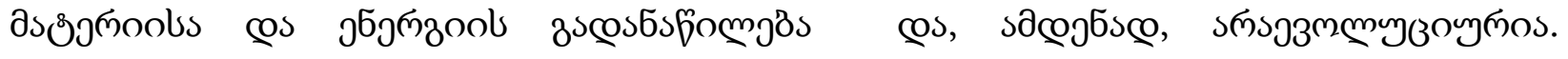

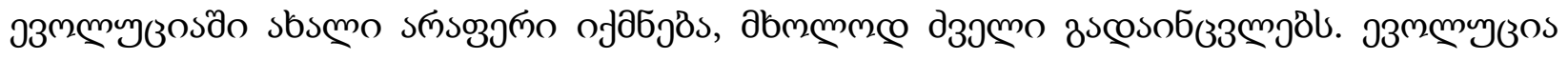

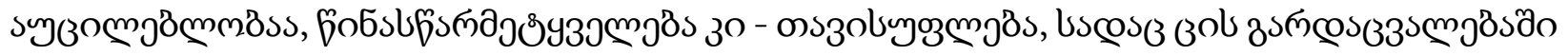




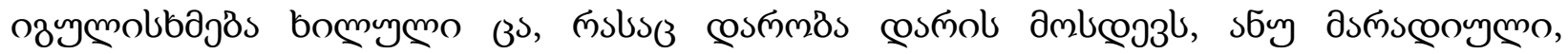

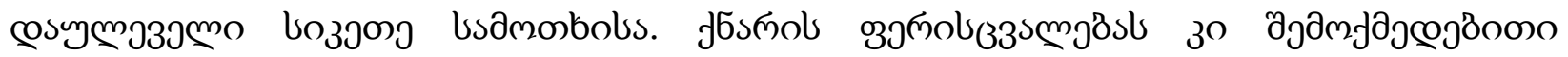

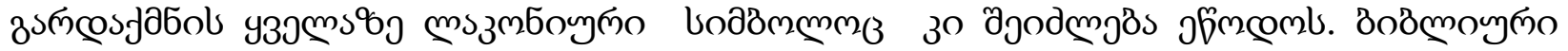

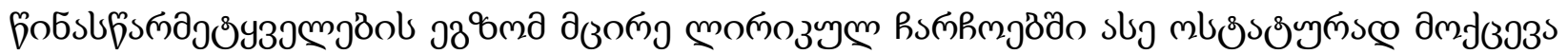

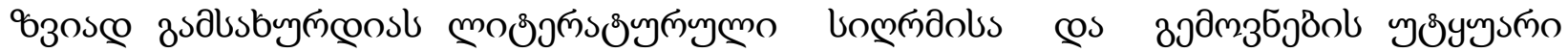

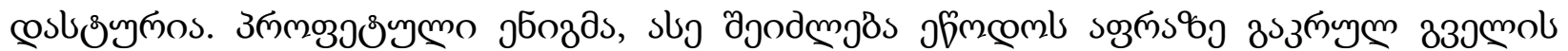

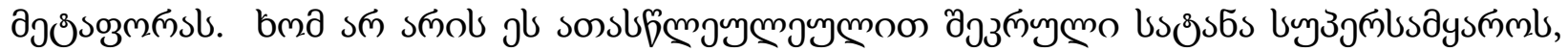

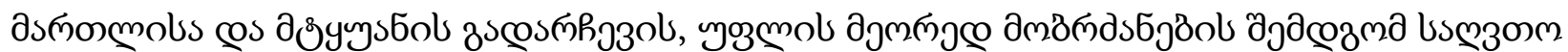

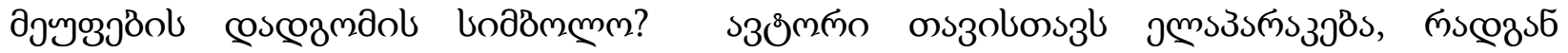

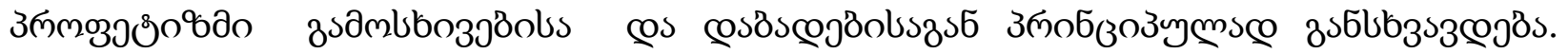

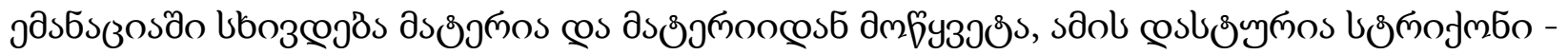

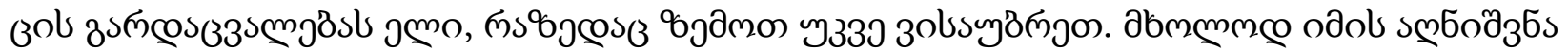

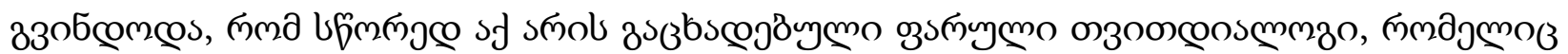

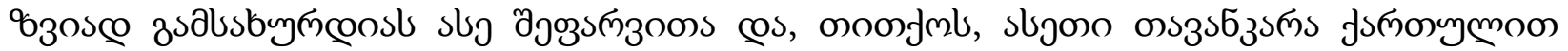

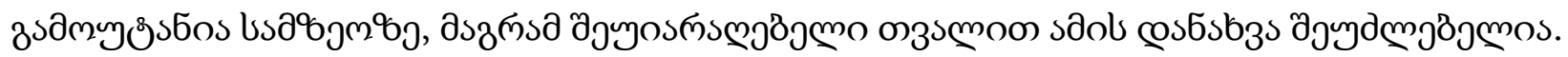

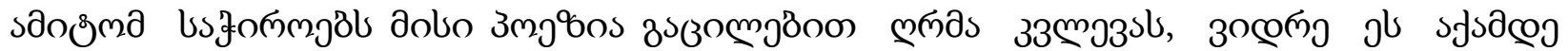

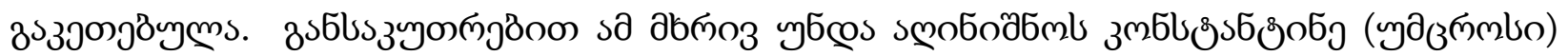

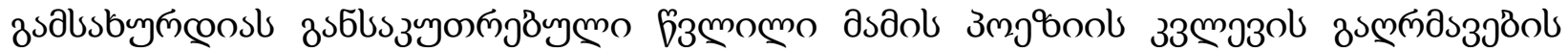

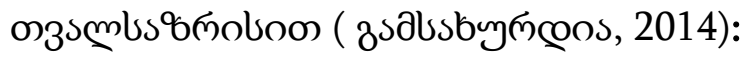

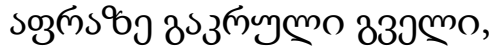

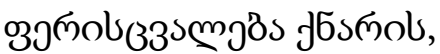

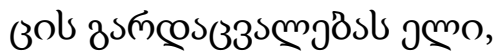

$$
\begin{aligned}
& \text { sbmms cosmmas casmol! }
\end{aligned}
$$

$$
\text { ( zsabsbym }
$$

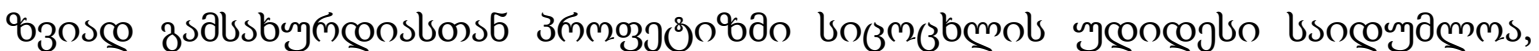

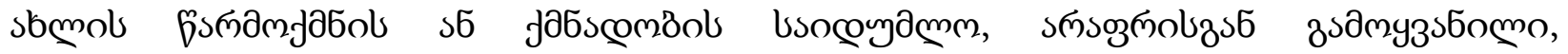

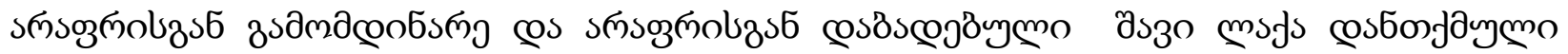

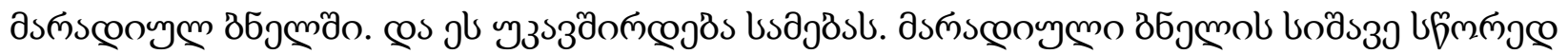

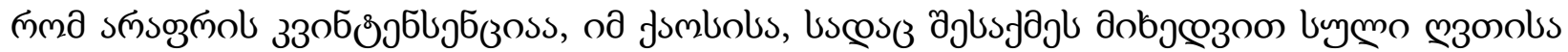

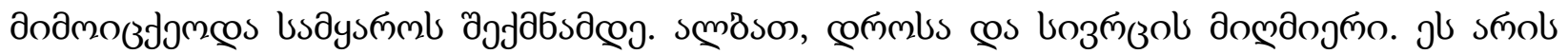
usoc

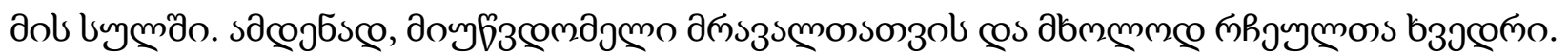

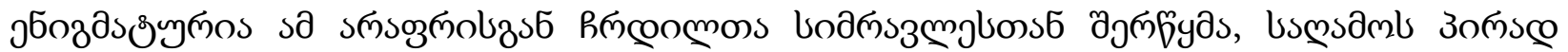

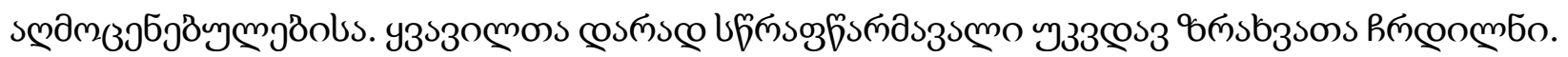

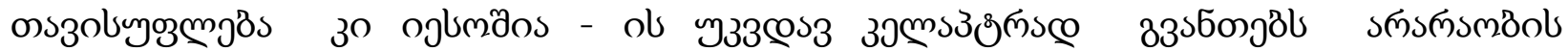

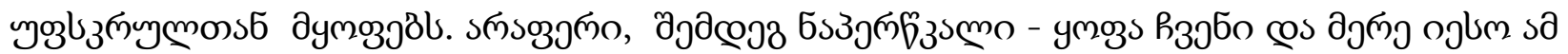

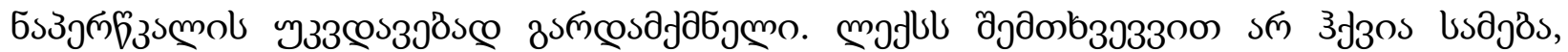

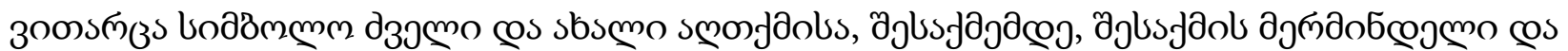

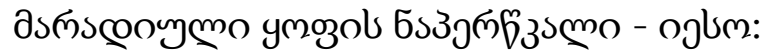

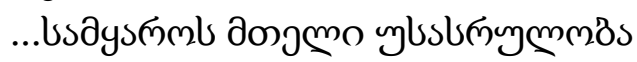

dszo msfs 


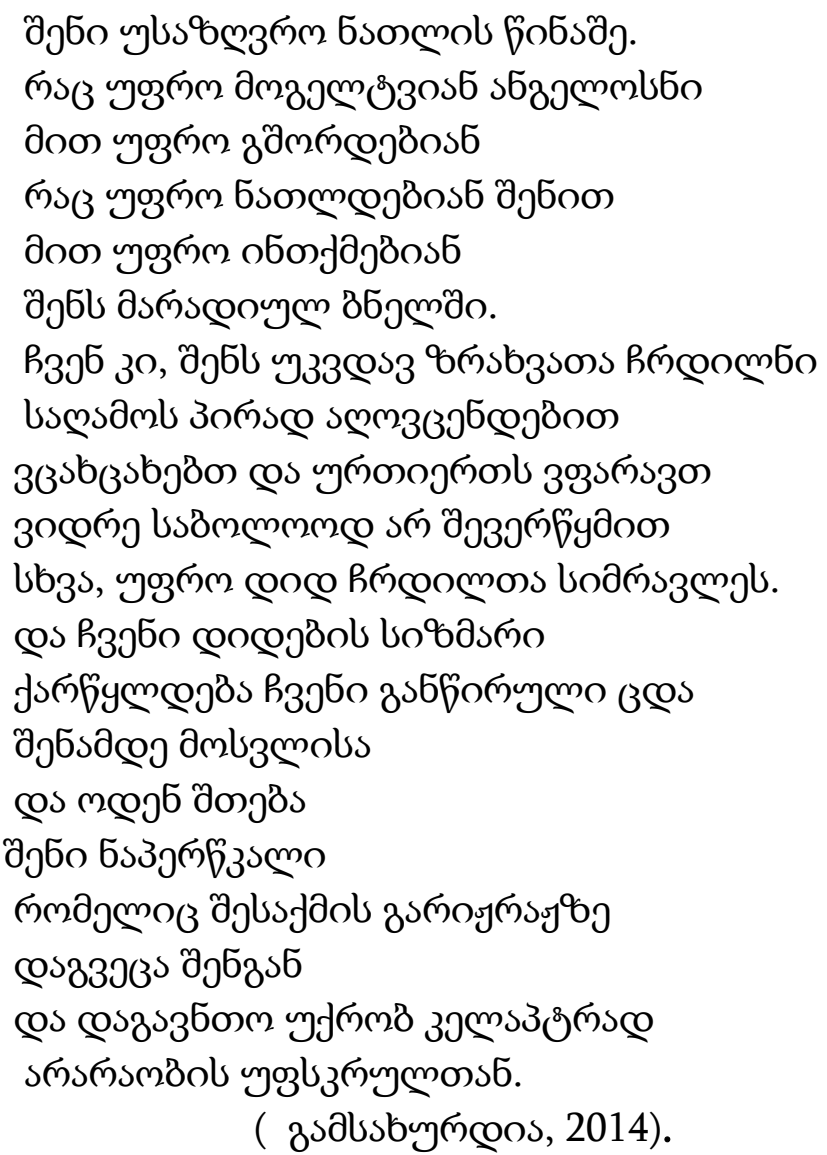

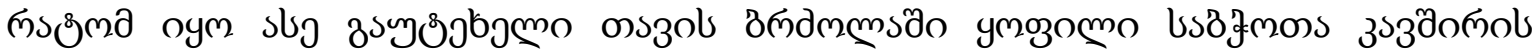

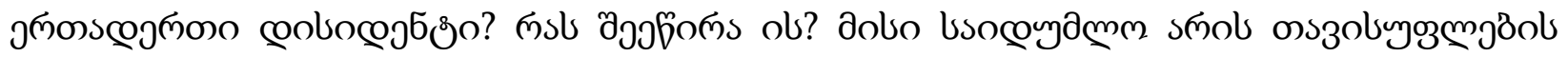

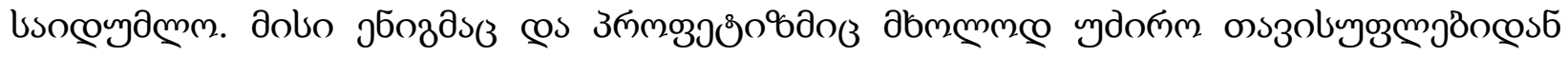

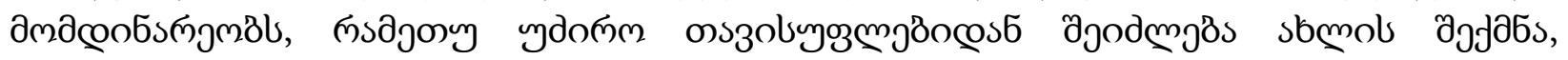

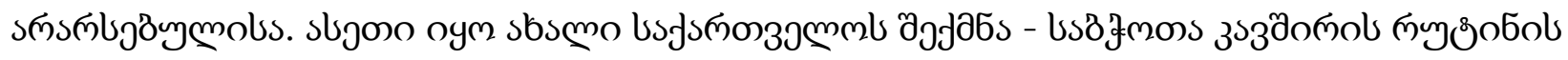

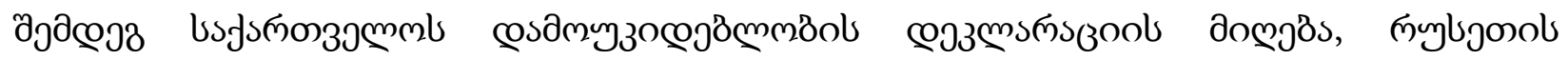

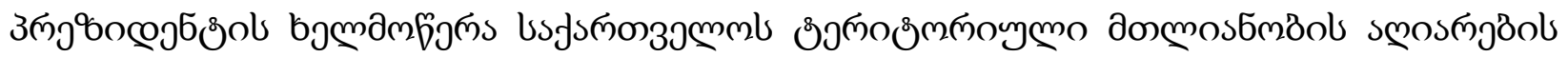

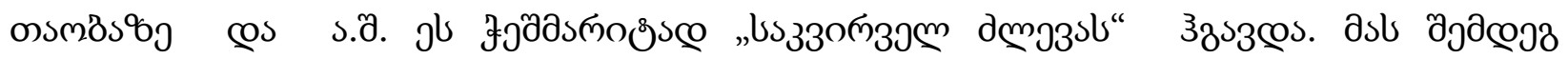

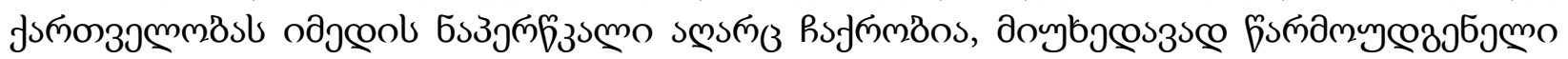

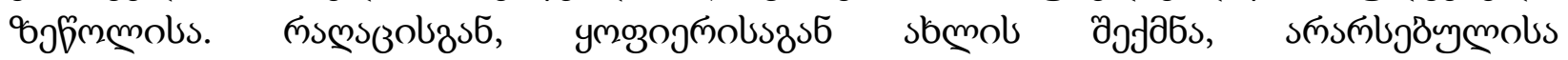

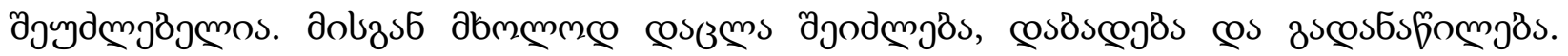

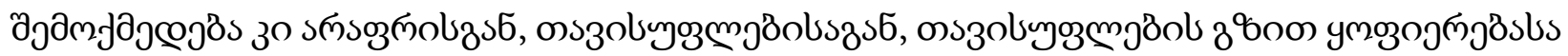

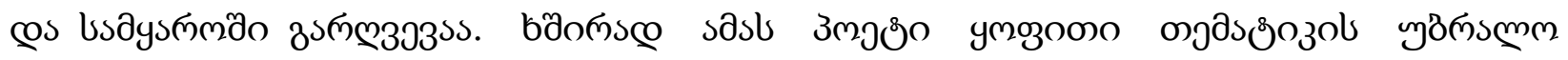

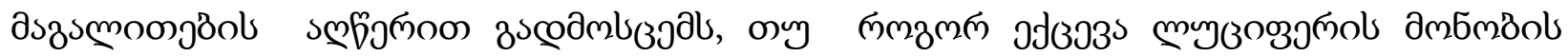

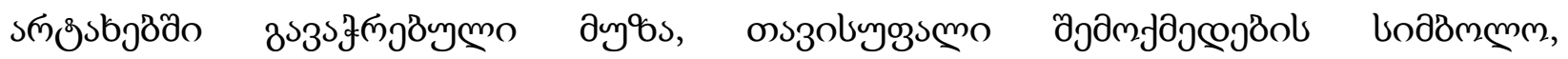

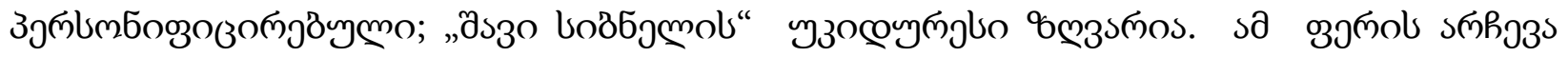

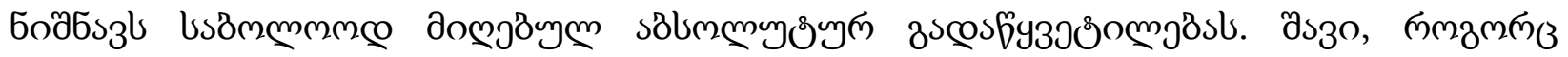

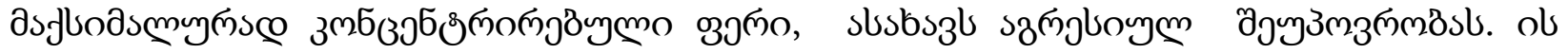

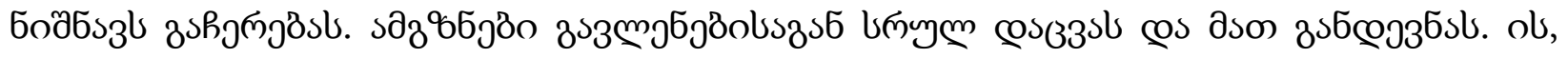

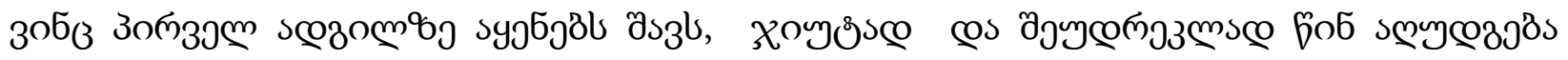




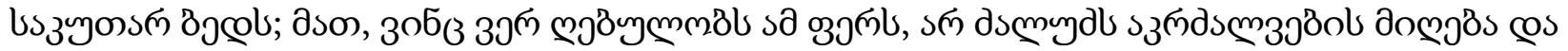

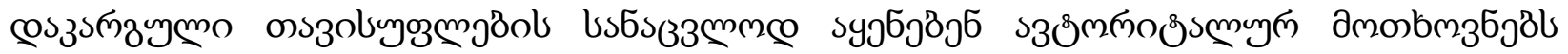

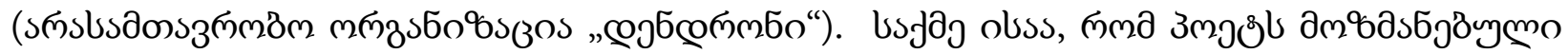

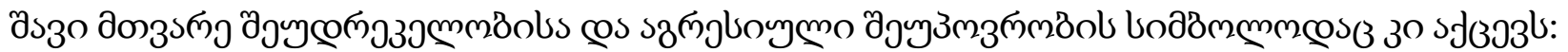

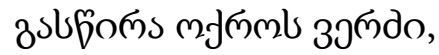

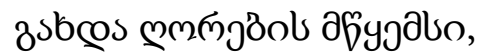

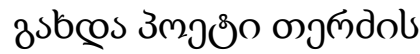
asfm, dsogo cos bjalso zscosozofys вэco, zstbœs ठsతిjöol dods, omRos bs

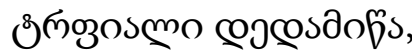

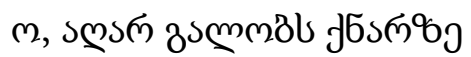

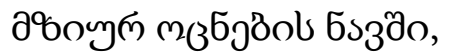

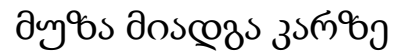
उ ҰЈल उल dson zo d39l 36 gmol y

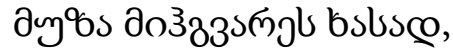

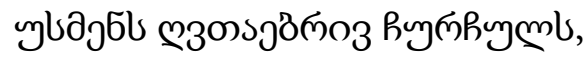

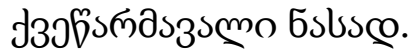
cos3xol cos з

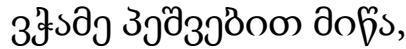

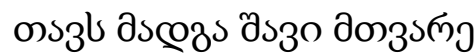
cos... увjo zsajmzods.

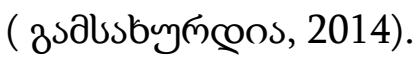

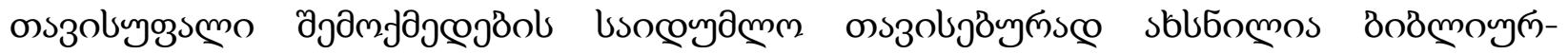

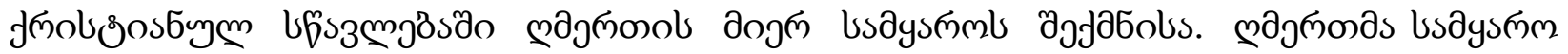

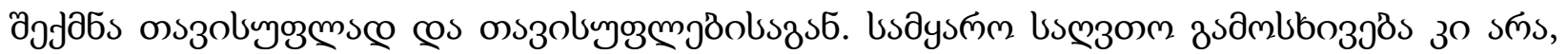

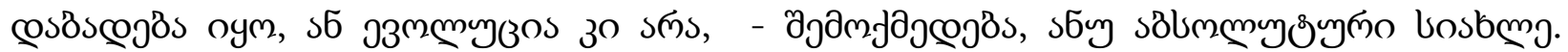

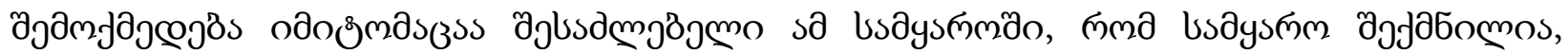

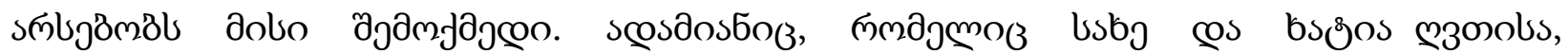

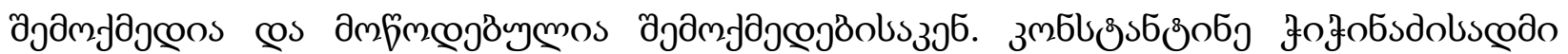

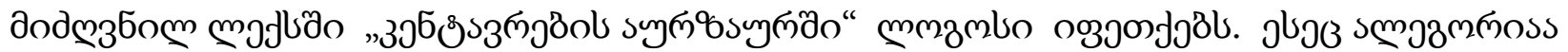

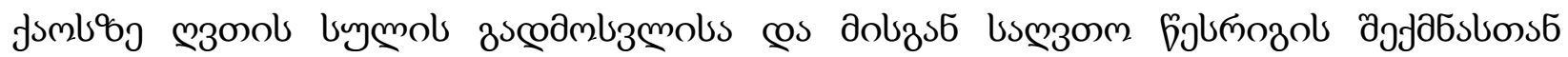

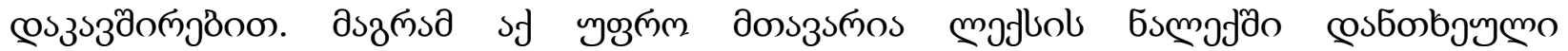

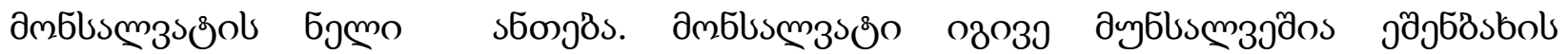

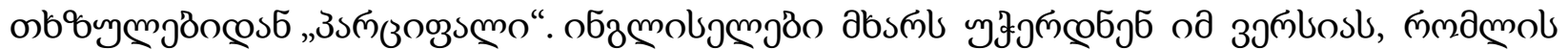

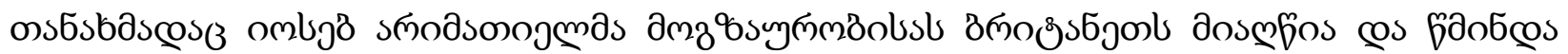

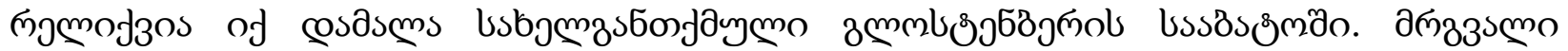

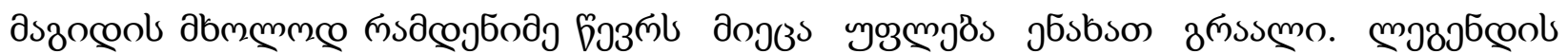

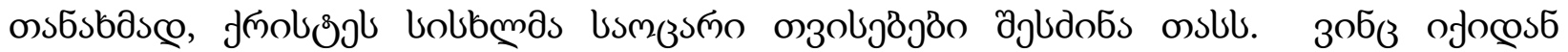




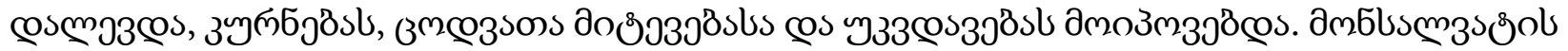

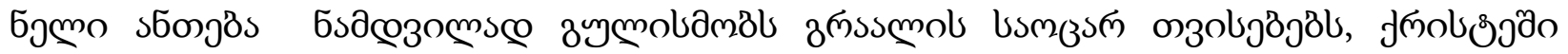

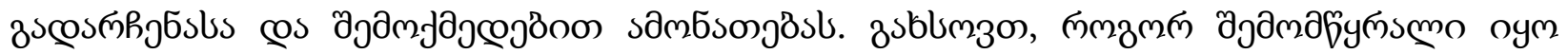

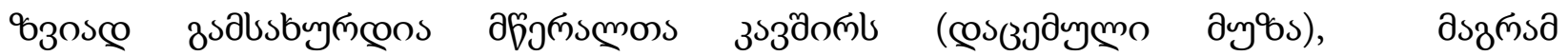

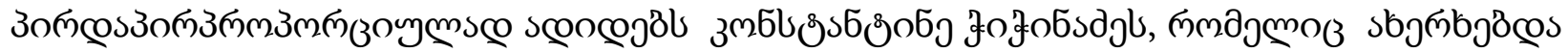

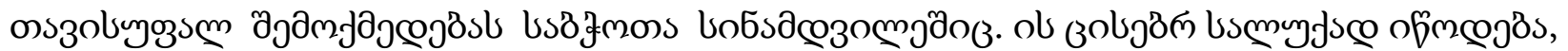

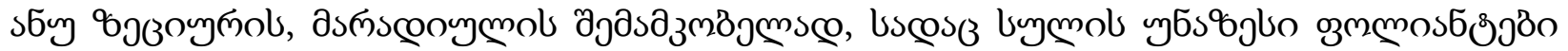

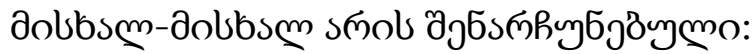

$$
\begin{aligned}
& \text {....es djamzjlas } \\
& \text { उјб } \\
& \text { asव̃o6 mмzmlas }
\end{aligned}
$$

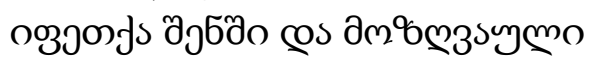

$$
\begin{aligned}
& \text { cosobonbs maflo } \\
& \text { cos sa 6smojôo }
\end{aligned}
$$

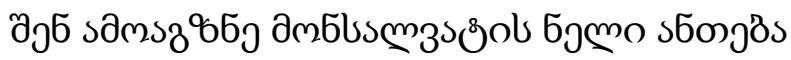

$$
\begin{aligned}
& \text { cos doo cosmuyds } \\
& \text { उolgin us muydo }
\end{aligned}
$$

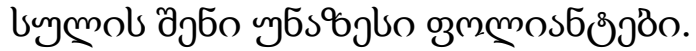

$$
\begin{aligned}
& \text { ( asalsbuynূ̣os, 2014). }
\end{aligned}
$$

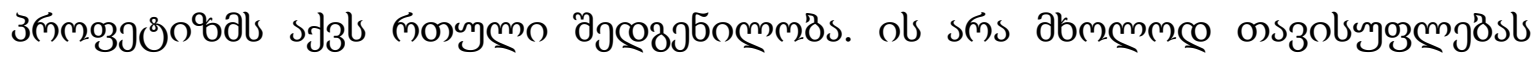

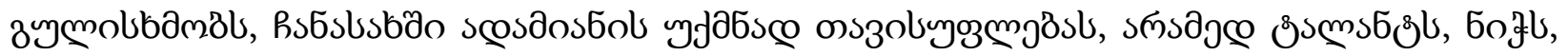

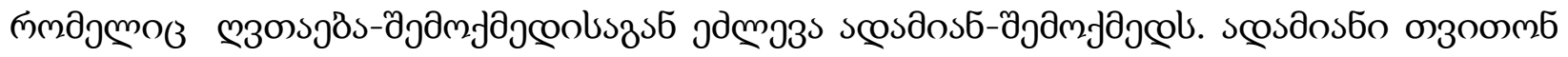

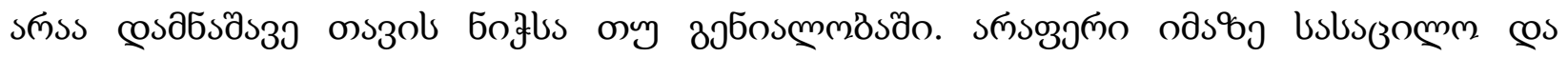

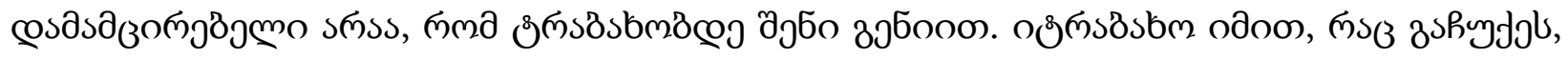

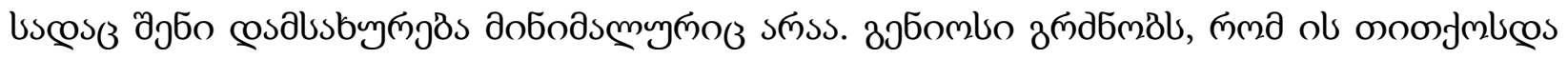
пзоомб зо зп дмдале

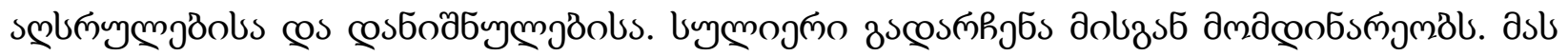

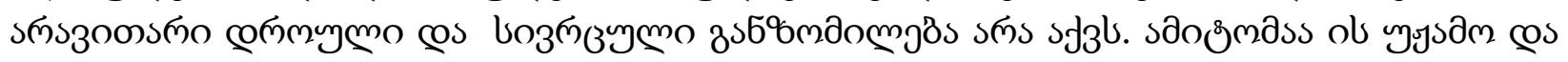

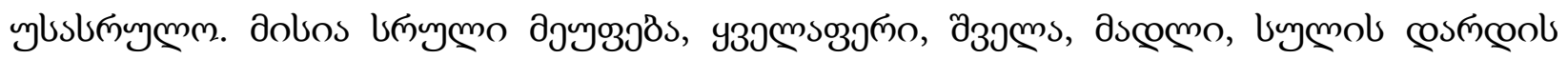
zs

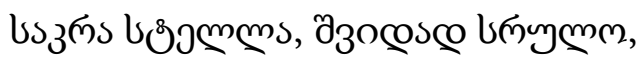

$$
\begin{aligned}
& \text { дjб }
\end{aligned}
$$

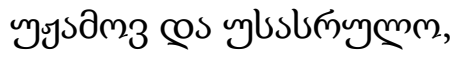

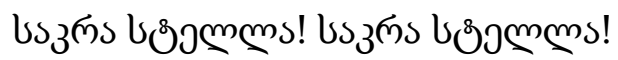

$$
\text { ( asalsbuymeos, 2014). }
$$

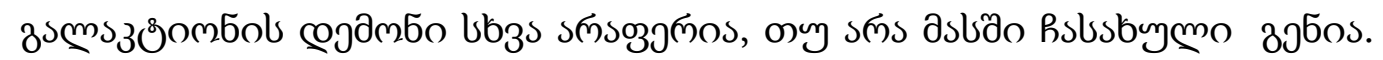

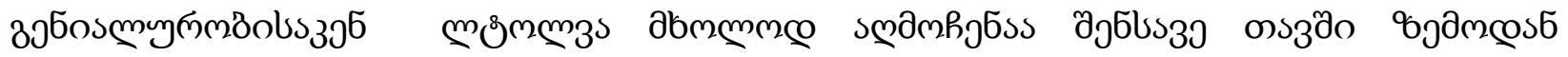

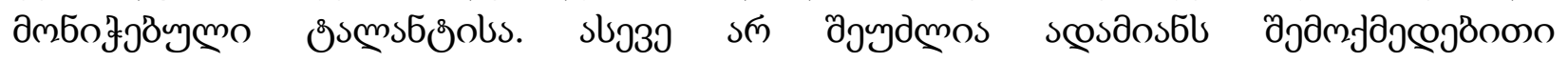

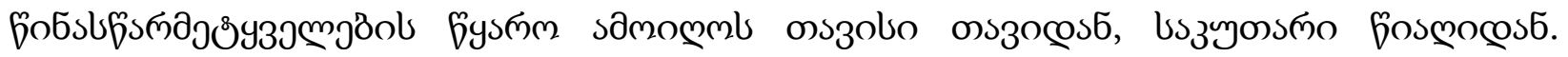

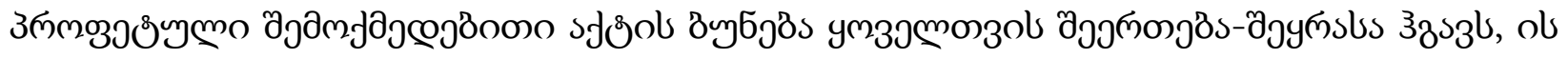

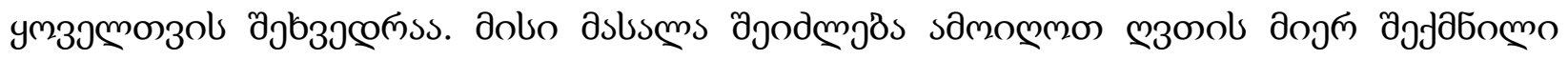




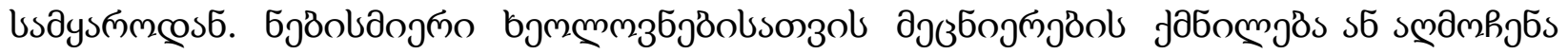

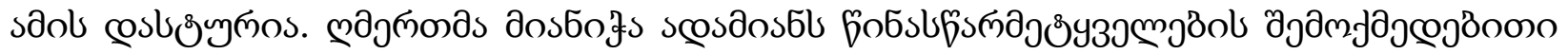

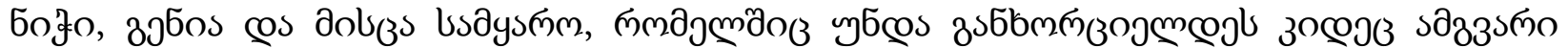

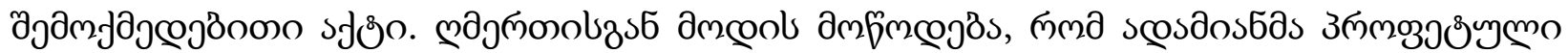

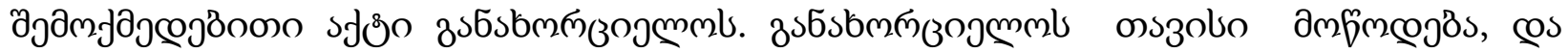

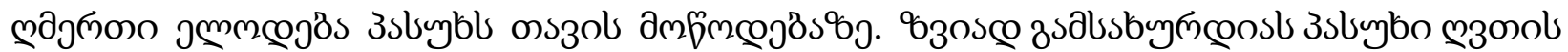

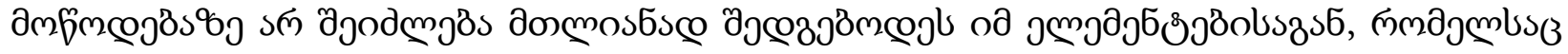

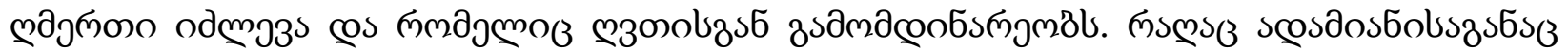

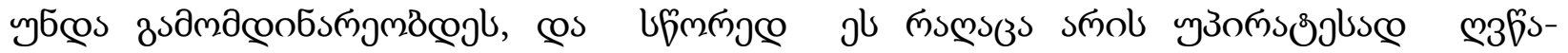

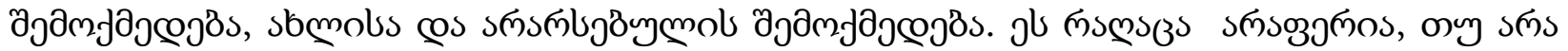

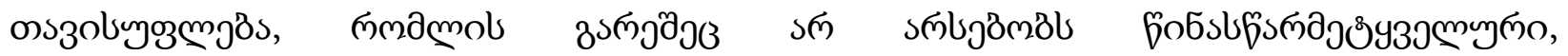

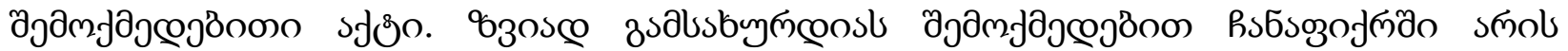

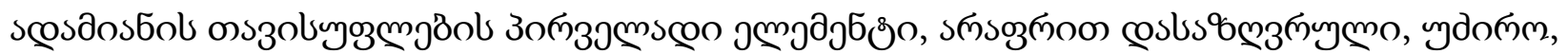

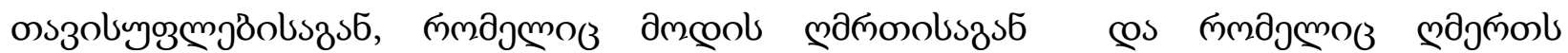

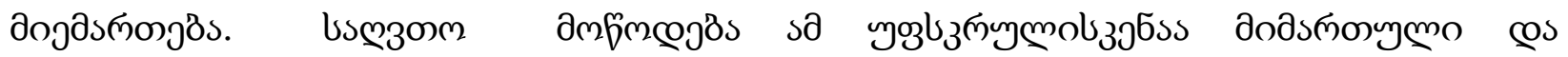

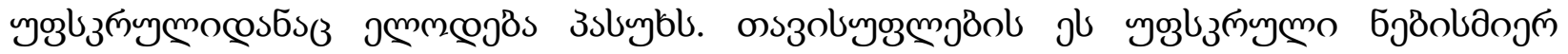

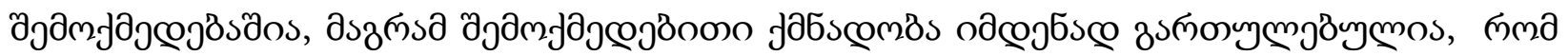

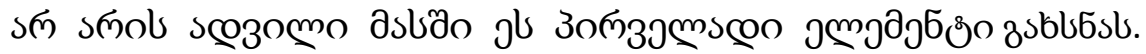

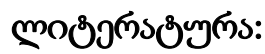

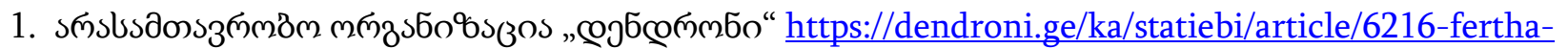
$\underline{\text { simbolika }}$

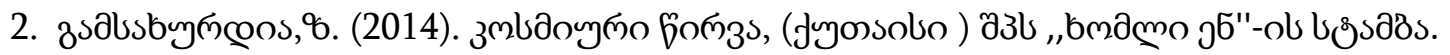

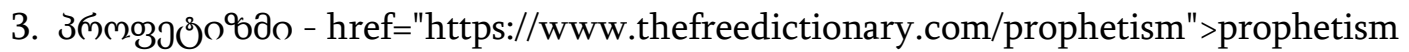

\section{Prophetism and Enigma in Zviad Gamsakhurdia's poetry}

Qiria Nana

Tbilisi No. 1 Experimental Public School Consultant of the National Center for Professional Development of Teachers

\begin{abstract}
Even a glance at Zviad Gamsakhurdia's work shows that he has experienced a long
\end{abstract}


creative burn, he is extremely strong in worldviews and eternal, unchanging, even strangely calm, but thematically changeable.

In his work, the arousal of feelings and the veil of thought are harmoniously combined. When the register of such harmony is very high, elements of prophethood appear in Zviad Gamsakhurdia's work and it must be said that his prophecy is infallible and skillfully placed in the artistic mold. In his poetry there is a skillfully blended vague allegory conveyed with a premeditated intent, something between mediocre openness and obscure, transparent and obscure. It can be boldly said that Zviad Gamsakhurdia is a master of enigmatic poetry.

The aim of the research is to critically analyze the poetry of the great Georgian figure, thinker and writer Zviad Gamsakhurdia (from a prophetic and enigmatic point of view).

The method is comparative. The merits and weaknesses of Zviad Gamsakhurdia's works can be seen in comparison, more - the first.

Research result - Prophecy by Zviad Gamsakhurdia is the biggest secret of life. Both his enigma and prophecy stem from bottomless freedom.

Key words: Prophecy, Enigma, Poetry, Gamsakhurdia, Creativity. 